\title{
Evitando urgências odontológicas em pacientes oncológicos: relato de caso
}

\author{
Avoiding dental urgencies in cancer patients: a case report \\ Evitar las urgencias dentales en pacientes con cáncer: reporte de un caso
}

\section{Resumo}

As manifestações orais do câncer são significativas tanto as decorrentes da doença quanto dos tratamentos antineoplásicos instituídos, pois podem comprometer a função, a estética e a qualidade de vida destes doentes, ou mesmo interromper os tratamentos. Objetivou-se, neste estudo, relatar um caso clínico de paciente diagnosticado com câncer em região de cabeça e pescoço submetido a tratamento antineoplásico bimodal quimio-radioterápico e descrever quais medidas devem ser tomadas para evitar as urgências odontológicas nestes pacientes. Paciente do gênero masculino, 53 anos, etilista e tabagista, apresenta diabetes mellitus tipo II, diagnosticado com carcinoma epidermóide moderadamente diferenciado em região supraglótica; encaminhado ao serviço de odontologia do Centro de Oncologia do Hospital Universitário Oswaldo Cruz da Universidade de Pernambuco previamente ao início do tratamento do câncer. Verificou-se a necessidade de adequação bucal e foi estabelecido protocolo operacional padrão de cuidados orais para indivíduos sob terapia antineoplásica. Após algumas semanas de tratamento o paciente apresentou complicações orais, com isso, fez-se um ajuste no protocolo, sendo instituído o protocolo operacional para o tratamento de necrose óssea. Assim, rapidamente a mucosa oral foi recuperada e as demais áreas da boca se mantiveram íntegras. Este relato corrobora com a literatura ao evidenciar que o acompanhamento odontológico do paciente oncológico é primordial para minimizar ou até mesmo inibir possíveis complicações decorrentes dos tratamentos, sendo essencial para evitar urgências odontológicas, favorecer o cuidado integral e humanizado oncológico, bem como a qualidade de vida do paciente.

Palavras-chave: Assistência odontológica; Câncer; Complicações bucais; Manifestações orais.

\begin{abstract}
The oral manifestations of cancer are significant, both resulting from the disease and from the antineoplastic treatments instituted, as they can compromise the function, aesthetics and quality of life of these patients, or even interrupt the treatments. The aim of this study was to report a clinical case of a patient diagnosed with cancer in the head and neck region submitted to bimodal chemotherapy and chemotherapy and describe what measures should be taken to avoid dental urgencies in these patients. A 53-year-old male patient, alcoholic and smoker, presents type II diabetes mellitus, diagnosed with moderately differentiated squamous cell carcinoma in the supraglottic region; referred to the dental service of the Oncology Center of the University Hospital Oswaldo Cruz of the University of Pernambuco prior to the start of cancer treatment. There was a need for oral adequacy and a standard operating protocol for oral care was established for individuals undergoing anticancer therapy. After a few weeks of treatment, the patient presented oral complications, with this, the protocol was adjusted, and the operational protocol for the treatment of bone necrosis was instituted. Thus, the oral mucosa was quickly recovered and the other areas of the mouth remained intact. This report corroborates the literature by showing that the dental follow-up of cancer patients
\end{abstract}


is essential to minimize or even inhibit possible complications resulting from the treatments, being essential to avoid dental urgencies, promote comprehensive and humanized cancer care, as well as quality of life of the patient.

Keywords: Dental care; Cancer; Bucal complications; Oral manifestations.

\begin{abstract}
Resumen
Las manifestaciones bucales del cáncer son significativas, tanto derivadas de la enfermedad como de los tratamientos antineoplásicos instaurados, ya que pueden comprometer la función, estética y calidad de vida de estos pacientes, o incluso interrumpir los tratamientos. El objetivo de este estudio fue reportar un caso clínico de un paciente diagnosticado de cáncer en la región de cabeza y cuello sometido a quimioterapia y quimioterapia bimodal y describir qué medidas se deben tomar para evitar urgências dentales en estos pacientes. Paciente varón de 53 años, alcohólico y fumador, que presenta diabetes mellitus tipo II, diagnosticada de carcinoma epidermoide moderadamente diferenciado en región supraglótica; remitido al servicio odontológico del Centro de Oncología del Hospital Universitario Oswaldo Cruz, Universidad de Pernambuco previo al inicio del tratamiento oncológico. Era necesaria la adecuación oral y se estableció un protocolo operativo estándar para el cuidado bucal para las personas que se sometían a terapia contra el cáncer. A las pocas semanas de tratamiento el paciente presentó complicaciones bucales, con ello se ajustó el protocolo y se instituyó el protocolo operativo para el tratamiento de la necrosis ósea. Así, la mucosa oral se recuperó rápidamente y las otras áreas de la boca permanecieron intactas. Este informe corrobora la literatura al mostrar que el seguimiento odontológico de los pacientes oncológicos es fundamental para minimizar o incluso inhibir las posibles complicaciones derivadas de los tratamientos, siendo fundamental para evitar urgencias dentales, promover la atención oncológica integral y humanizada, así como la calidad de vida del paciente.
\end{abstract}

Palabras clave: Cuidado dental; Cáncer; Complicaciones bucales; Manifestaciones orales.

\title{
1. Introdução
}

O câncer é considerado um problema de saúde pública mundial, e está entre as quatro principais causas de morte antes dos 70 anos de idade na maioria dos países (Instituto Nacional de Câncer José Alencar Gomes da Silva [INCA], 2019); se configura pelo crescimento desordenado das células que se dividem de forma rápida, agressiva e incontrolável, acarretando no desenvolvimento de tumores que afetam tecidos e órgãos do corpo (Arantes et al., 2020). É uma doença de alta incidência mundial e o Brasil tem um dos índices mais altos do mundo (Domingos et al., 2014). De acordo com a estimativa do INCA (2019), para o Brasil, haverá entre 2020-2022 a ocorrência de 625 mil casos novos de câncer, para cada ano. O aumento da incidência e mortalidade do câncer tem ocorrido pelo envelhecimento, crescimento populacional, mudança na distribuição e na prevalência dos fatores de risco do câncer.

As neoplasias malignas em região de cabeça e pescoço são a $6^{\circ}$ mais prevalente (Borges et al., 2018), representando cerca de 5\% de todas as neoplasias existentes, atingindo em torno de 1,7\% da população brasileira (Arantes et al., 2020). Conforme o INCA (2019) o câncer de cabeça e pescoço compreende o quinto câncer que mais acomete homens e décimo primeiro em mulheres e engloba os tumores localizados na pele e lábios, cavidade oral, orofaringe, laringe, hipofaringe, nasofaringe, glândulas salivares, cavidade nasal e seios paranasais, meato acústico externo e ouvido médio (Arantes et al., 2020).

Os recentes avanços no tratamento do câncer levaram a um aumento na sobrevida, assim como mudanças na incidência, natureza e gravidade das complicações bucais, afetando a qualidade de vida desses indivíduos. Assim sendo, tornou-se cada vez mais reconhecida a necessidade do acompanhamento odontológico destes pacientes, além de se estabelecer cuidados bucais específicos para eles, objetivando garantir a saúde bucal e o bem-estar geral destes (Ghelardi et al., 2008).

Segundo Osterne et al. (2008), muitos pacientes depois de iniciado o tratamento de quimioterapia e radioterapia em região de cabeça e pescoço apresentam risco de desenvolvimento de lesões bucais dentárias ou periodontais, relacionadas a focos odontogênicos preexistentes. Uma vez que, a falta de uma dieta balanceada, higiene bucal deficiente, presença de dentes fraturados ou restaurações defeituosas, restos radiculares, doença periodontal ou próteses mal ajustadas podem contribuir para o desenvolvimento de infecções locais ou sistêmicas que, por sua vez, podem impedir a administração de terapia antineoplásica completa, exigindo redução na dose ou atraso na aplicação da terapia (Vidal, 2015). 
Este artigo se propõe a relatar um caso clínico de paciente diagnosticado com câncer em região de cabeça e pescoço sob tratamento antineoplásico e determinar os procedimentos odontológicos que podem contribuir para evitar urgências odontológicas em pacientes oncológicos sob terapias antineoplásicas, ou após elas.

\section{Revisão de Literatura}

A Urgência consiste em uma situação, na qual é necessária uma intervenção rápida, visando minimizar as complicações e o sofrimento do paciente, em que não há o risco iminente de morte (Bezerra et al., 2015). Na urgência odontológica é necessária a adoção de medidas com o intuito de aliviar os sintomas dolorosos, infecciosos e estéticos da cavidade bucal (Queiroz et al., 2019) e as doenças mais frequentes são as que acometem a polpa dentária e o periodonto (Bezerra et al., 2015). Com o quadro clínico de dor, o paciente pode ter suas atividades cotidianas limitadas, gerando um impacto negativo na qualidade de vida. Consequentemente, pode haver uma diminuição da autoconfiança pessoal, repercutindo de forma negativa nas relações sociais, econômicas, educacionais e ocupacionais (Queiroz et al., 2019).

A falta de higiene bucal e a falta de acompanhamento periódico de um cirurgião dentista são fatores que podem desencadear uma urgência odontológica (Bezerra et al., 2015). Em vista disso, para que se desenvolva estratégias de atendimento odontológico e programas de promoção de saúde que sejam eficientes é de suma importância que se tenha conhecimento científico de como as doenças da cavidade oral se instalam, sua progressão e ainda dados epidemiológicos sobre sua prevalência, severidade e distribuição na comunidade (Figueiredo \& Nogueira, 2013).

Perante o exposto, é estimado que cerca de $40 \%$ dos pacientes que durante o tratamento fazem uso de quimioterapia e radioterapia, irão desenvolver complicações orais devido a estomatotoxicidade direta ou indireta (Floriano et al., 2017).

Para o tratamento dos pacientes diagnosticados com câncer, a escolha do método de tratamento será realizada de acordo com a natureza e extensão do tumor e compreende a cirurgia, a radioterapia e a quimioterapia combinadas ou separadamente (Floriano et al., 2017). Cada uma dessas terapias atua de forma distinta, mas todas apresentam algum potencial para causar efeitos colaterais na cavidade oral. A cirurgia pode acarretar problemas funcionais e estéticos (Macedo et al., 2019). A radioterapia e a quimioterapia atuam no processo de divisão celular, inibindo o crescimento e a proliferação das células. Conduto, essas abordagens não são seletivas, podendo desenvolver reações adversas que se manifestam com alta incidência no epitélio oral, devido à rapidez e a frequência da renovação celular comprometendo a integridade e função da cavidade oral (Macedo et al., 2019; Neves, 2018;).

O tipo e o grau de malignidade do tumor, a dose das drogas utilizadas, a duração da quimioterapia, a idade e o nível de higiene oral, antes e durante a terapia antineoplásica, são fatores que irão determinar a severidade das complicações bucais (Hespanhol et al., 2010). Com isso, compreender de forma correta os sinais e sua correlação com sintomas e drogas ou radiação utilizadas nos tratamentos oncológicos tornam as complicações orais mais previsíveis, o que pode facilitar a sua prevenção e seu tratamento, sendo de suma importância a integração da odontologia na equipe médica de oncologia (Floriano et al., 2017).

Os efeitos colaterais orais são divididos em agudos: ocorrem durante ou no pós-imediato do tratamento ou crônicos: ocorrem meses ou anos após o tratamento, em que os agudos são os mais prevalentes na região bucal, sendo eles: infecções oportunistas, mucosite oral, xerostomia, disgeusia e disfagia (Neves, 2018).

Os pacientes irradiados na região de cabeça e pescoço apresentam risco aumentado para candidíase, provavelmente em decorrência da redução do fluxo salivar e consequente redução da atividade fagocítica dos granulócitos salivares contra estes microorganismos. Pode ocorrer na forma de placas pseudomembranosas, áreas eritematosas, atrofia crônica e queilite angular (Vidal, 2015). As manifestações clínicas da mucosite oral são caracterizadas pelo desenvolvimento de uma coloração 
esbranquiçada da mucosa, com uma sensação de queimação, perda da camada de queratina seguida da exposição da mucosa atrófica, podendo ocorrer áreas de ulceração com a formação de uma membrana fibrinopurulenta, amarelada e removível, nesse estágio o paciente refere dor localizada, odinofagia e disfagia (Tosin, 2017).

A Xerostomia se configura pela sensação subjetiva de secura da cavidade bucal, devido a redução qualitativa e quantitativa do fluxo salivar pela exposição das glândulas salivares maiores ao campo de radiação. A diminuição do fluxo salivar pode ser afetada pela cirurgia, radioterapia e quimioterapia, influenciando nos seguintes fatores: capacidade tampão, aumentando os níveis de desmineralização do esmalte dental; na quantidade de mucina, deixando a mucosa desprovida de proteção, e na sua propriedade de lubrificação. Também sofrem alteração diversos eletrólitos, como cálcio, potássio, sódio e fosfato, ficando os indivíduos mais susceptíveis ao desenvolvimento da doença periodontal, cáries rampantes, infecções bucais fúngicas e bacterianas (Vidal, 2015; Tosin 2017).

A Cárie de radiação pode ser considerada um efeito secundário da xerostomia, com a diminuição da capacidade imunológica antibacteriana presente na saliva (Tosin, 2017). Além disso, a radiação atua sobre os odontoblastos, provocando a diminuição na formação de dentina reacional, tornando o esmalte dentário mais vulnerável a cárie (Vidal, 2015). As cáries de radiação podem surgir nos primeiros 3 meses após o tratamento radioterápico. Por isso, o foco do tratamento odontológico deve estar na prevenção (Duarte et al., 2019). Umas das complicações orofaciais mais graves é a osteorradionecrose (Tosin, 2017), pois a radiação torna o tecido hipóxico, hipovascularizado e hipocelulado, com a perda da mucosa de revestimento bucal e consequente exposição do tecido ósseo necrótico (Vidal, 2015).

É importante avaliar a condição oral inicial e o possível impacto dessa condição na qualidade de vida de pacientes com câncer, pois existe uma condição precária na saúde bucal do adulto e população idosa no Brasil, a maioria dos pacientes apresentam uma alta prevalência de cárie, doenças periodontais, patologias, próteses mal ajustadas e uma higiene oral precária. De uma forma geral, os pacientes oncológicos apresentam necessidades odontológicas significativas que implicam em uma abordagem odontológica prévia ao tratamento oncológico, assim como, durante e após o tratamento para que se possa evitar e controlar as complicações que venham a surgir. Portanto, doenças dentárias pré-existentes juntamente com a higiene oral inadequada, são fatores desencadeantes para complicações orais advindas do tratamento oncológico (Calmon et al., 2019; Vieira et al., 2012).

\section{Metodologia}

Trata-se de um relato de caso clínico de um paciente diagnosticado com câncer em região de cabeça e pescoço e descrição das medidas adotadas para evitar, controlar e tratar complicações buco-dentais. Estudo realizado no Serviço de Odontologia do Centro de Oncologia do Hospital Universitário Oswaldo Cruz da Universidade de Pernambuco CEON/HUOC/UPE e mediante os esclarecimentos, o paciente assinou o Termo de Consentimento Livre e Esclarecido, consentindo a divulgação de seu caso para fins acadêmicos. O relato de caso integra projeto de pesquisa aprovado pelo Comitê de Ética em Pesquisa da Universidade de Pernambuco, sob o parecer nº. 3.184.856 (Cândido et al., 2021).

Consoante a literatura (Albrecht et al., 2005; Estrela, 2018; Lakatos \& Marconi, 2003; Pereira et al., 2018) um caso para ser relatado, investigado, analisado e discutido precisa ter alguma particularidade que o diferencie, tem que ser especial. Um bom relato de caso deve ter o objetivo de acrescentar benefícios às práticas atuais ou de traçar possíveis novas direções na pesquisa de determinado tema em que um único ou poucos indivíduos possam ser representativos, destacam Albrecht et al. (2005), tal qual segue descrito neste cuidadoso e detalhado relato de um caso clínico. Quanto aos possíveis riscos e desconfortos, de acordo com a Resolução 466/2012, de 12 de dezembro de 2012, sobre as Normas Regulamentadoras de Pesquisas envolvendo seres humanos, de origem física, psíquica, moral, intelectual, social, cultural ou espiritual, eles foram 
contornados e controlados através da valorização, preservação e sigilo das informações e privacidade do participante. Os benefícios esperados com o resultado desse relato é permitir uma melhor compreensão tanto do processo biológico quanto das repercussões agudas e/ou crônicas buco-dentais decorrentes tanto da doença quanto dos tratamentos antineoplásicos, sobre a necessidade do cuidado buco-dental especializado oncológico, bem como do cuidado buco-dental nas atenções primária e secundária em saúde.

\section{Relato de Caso}

Paciente do gênero masculino, 53 anos de idade, etilista e tabagista. Apresenta Diabetes Mellitus tipo II (DMII), nega histórico de Hipertensão Arterial Sistêmica (HAS), assim como histórico de câncer na família. Encaminhado ao Serviço de Cirurgia de Cabeça e Pescoço - CEON/HUOC/UPE, em agosto de 2018, apresentando quadro de disfagia (dificuldade para deglutir) e odinofagia (dor ao deglutir) há aproximadamente 3 meses, sem perda de peso importante. A biópsia foi realizada e o laudo histopatológico (AP: 501682-18) conclusivo para carcinoma epidermóide moderadamente diferenciado (Tumor ulcerado vegetante), Estádio clínico EII, T2N0M0, em região de supraglótica à esquerda, localizado na laringe. Foi instituído tratamento antineoplásico através de quimioterapia neoadjuvante semanal com carboxiplatina $(215 \mathrm{~g})+\operatorname{taxol}(135 \mathrm{~g}) 80 \mathrm{mg} / \mathrm{cm}^{2}$, totalizando 9 semanas; 35 sessões de radioterapia, com cisplatina semanal.

Previamente ao início do tratamento antineoplásico o paciente foi encaminhado ao serviço de Odontologia CEON/HUOC/UPE para adequação do meio bucal. Foi realizada a anamnese, exame físico extra e intraoral, e solicitada radiografia panorâmica dos maxilares. Foi identificado indivíduo parcialmente dentado, apresentando restos radiculares e cáries dentárias, confirmados radiograficamente (Figuras 1A, 1B e 2).

Figuras 1A, 1B. Condição clínica inicial do arco dental superior e inferior.

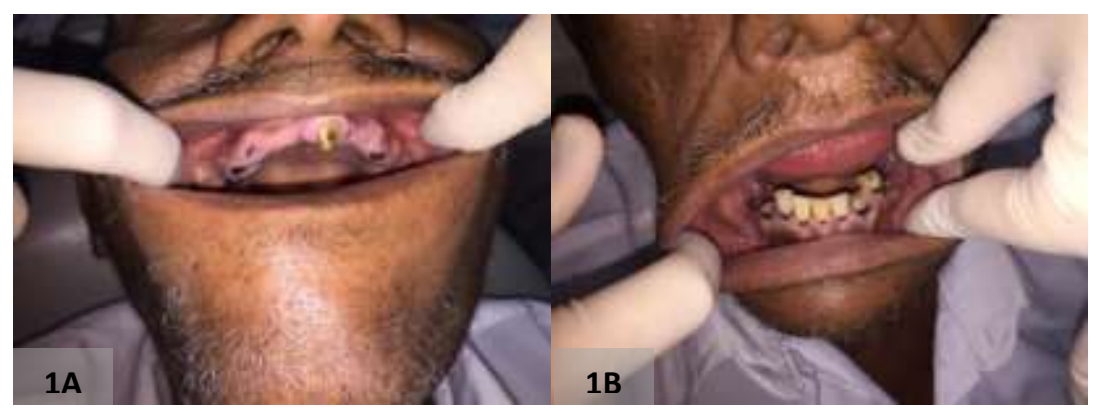

Fonte: Autores.

Figura 2. Radiografia Panorâmica dos Maxilares.

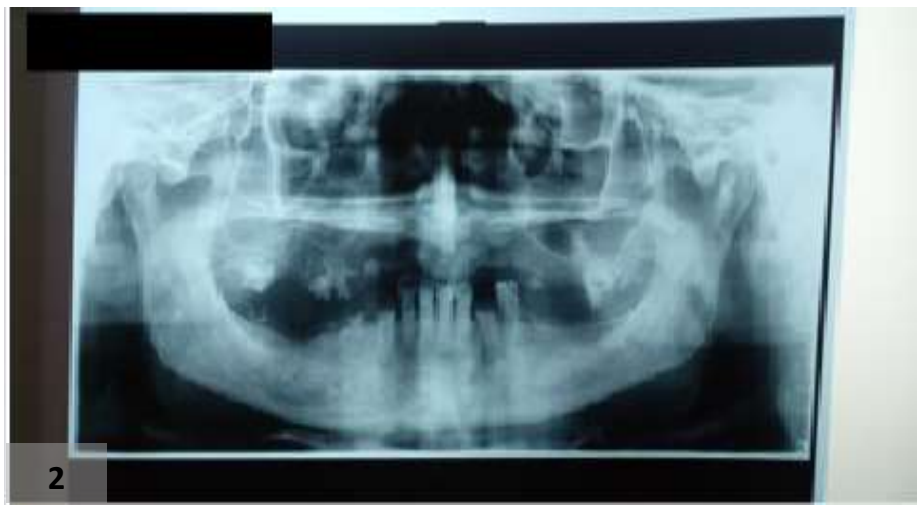

Fonte: Autores. 
Foi realizada a adequação do meio bucal através da remoção de focos de infecção, ou seja, exodontias múltiplas (Figura 3). Foi instituído o Protocolo Operacional Padrão de Cuidados Orais (POP - Oral) (VIDAL, 2009), para indivíduos sob terapia antineoplásica, sendo prescrito uso de escova dental com cabeça pequena e cerdas macias, creme dental não abrasivo (infantil), bochechos com solução oral de bicarbonato de sódio (8/8h), bochechos com digluconato de clorexidina $0,12 \%$ (12/12h), bochechos com solução oral de nistatina 100.000 UI (6/6h) e aplicação tópica de vitamina E (oleosa) 12/12h; associado a aplicação do Laser de Baixa Potência (LBP), aparelho MMO Laser Duo (MMOptics - São Carlos, SP, Brasil, emissor de luzGaA1As, comprimento de onda $660 \mathrm{~nm}$, potência de $100 \mathrm{~mW}$, energia de $2 \mathrm{~J}$ e área de feixe $3 \mathrm{~mm}^{2}$ ), aplicação por varredura, duas vezes na semana.

Figura 3. Dentes cariados e restos radiculares removidos para adequação do meio bucal.

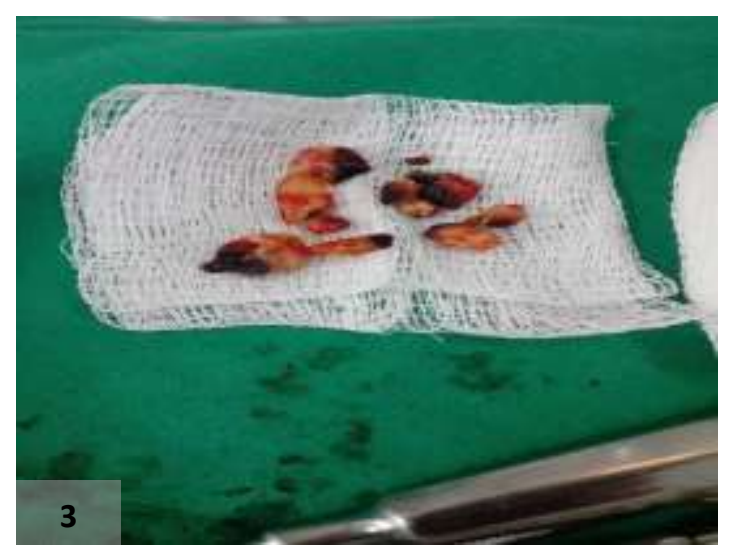

Fonte: Autores.

O paciente apresentou quadro de xerostomia, disgeusia, mucosite oral grau II (Escala OMS/WHO), e identificou em 4 (leve a moderada) a dor oral, na Escala Numérica da Dor. Foi detectada exposição óssea em rebordo alveolar superior esquerdo (Figura 4) na $23^{\circ}$ sessão de radioterapia, sendo instituído o Protocolo Operacional Padrão de Cuidados Orais (POP-Oral) para o tratamento de necrose óssea (VIDAL, 2009), através de bochechos diários com bicarbonato de sódio (8/8h), digluconato de clorexidina $0,12 \%(12 / 12 \mathrm{~h})$ e água oxigenada a 10 volumes (12/12h), associado à aplicação do LBP aparelho MMO Laser Duo (MMOptics - São Carlos, SP, Brasil, emissor de luzGaA1As, comprimento de onda 660nm, potência de 100mW, energia de 2J e área de feixe $3 \mathrm{~mm}^{2}$, e com espectro infravermelho, $880 \mathrm{~nm}$, com potência fixa de $100 \mathrm{~mW}$ e densidade de energia de $4 \mathrm{~J} / \mathrm{cm}^{2}$ ), aplicação pontual, duas vezes na semana. 
Figura 4. Exposição óssea identificada na $23^{\circ}$ sessão de radioterapia em rebordo alveolar superior esquerdo $+/-2 \mathrm{~mm}$.

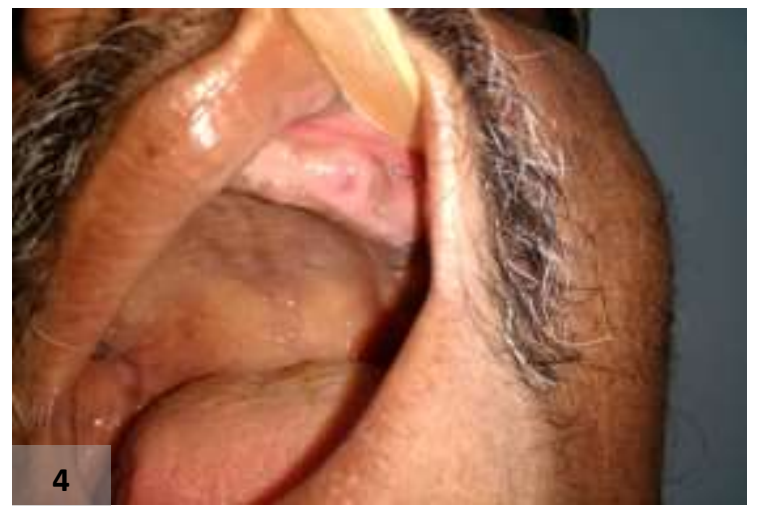

Fonte: Autores.

O paciente recuperou a mucosa oral, em 22 dias após o início do POP-Oral (Figura 5). As demais áreas da boca se mantiveram íntegras (Figuras 6A, 6B). O paciente seguiu sem queixa de dor ou desconforto oral, sob acompanhamento médico e multiprofissional, e, atualmente, aguarda a reabilitação oral protética.

Figura 5. Reparo tecidual da exposição óssea identificada na $23^{\circ}$ sessão de radioterapia, em rebordo alveolar superior esquerdo +/-2mm, após 22 dias do início do protocolo oral de cuidado de necrose óssea.

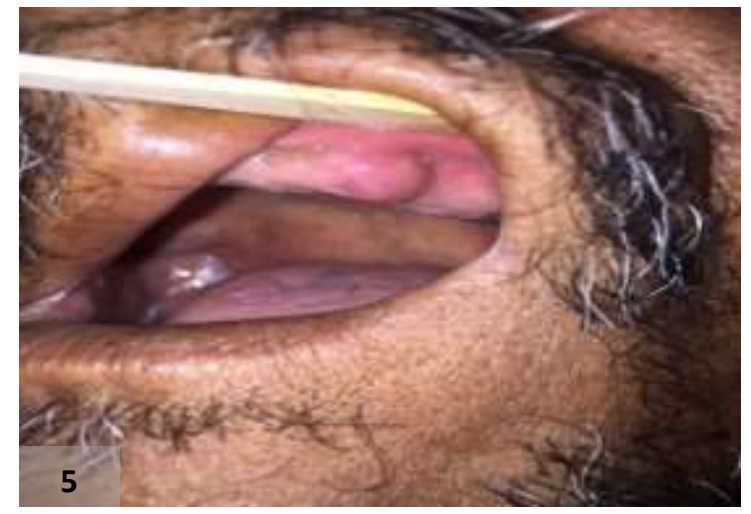

Fonte: Autores.

Figuras 6A, 6B. Demais regiões de mucosa intraoral íntegras durante o tratamento radioterápico, $35^{\circ}$ sessão de radioterapia.

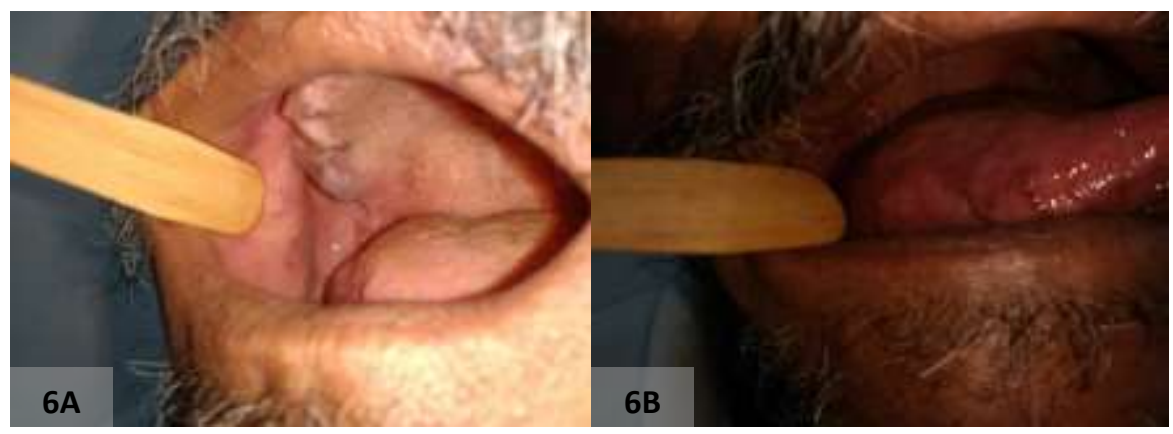

Fonte: Autores. 


\section{Discussão}

De acordo com o INCA (2019), o câncer de laringe pode acontecer em três regiões supraglote, glote e subglote e é um dos mais comuns que afetam a região da cabeça e pescoço. Tal qual relatado neste caso, e corroborado pela literatura, constatase que o câncer de laringe acomete principalmente homens acima de 40 anos e o tipo histológico mais encontrado, em mais de 90\% dos pacientes é o carcinoma de células escamosas, neoplasia de origem epitelial. Os principais fatores de risco incluem álcool, tabaco e quando combinados potencializam o desenvolvimento do câncer, além de outros fatores como excesso de gordura corporal e fatores relacionados a exposição ocupacional (INCA, 2019).

No decorrer da terapia antineoplásica focos de infecção preexistentes como dentes comprometidos, com bordas afiadas, fraturados ou restaurações defeituosas facilitam o acometimento da mucosa e o desenvolvimento de infecções podendo exacerbar os processos inflamatórios da polpa dentária (Macedo et al., 2019). Assim, faz-se necessário a adequação bucodental prévia.

Araújo et al. (2009), avaliaram a saúde bucal de pacientes com câncer em região de cabeça e pescoço, registrando índice de dentes cariados, perdidos e obturados (CPOD) igual a 26,8, onde o componente perdido foi responsável por 91,5\% do CPOD, e o componente cariado, por 5,31\%. O CPOD é um importante indicador para avaliar a prevalência da cárie dentária e suas sequelas. De acordo com a Organização Mundial de Saúde, os valores do CPOD médio para dentição permanente são: 0 a 1,1 muito baixa; 1,2 a 2,6 baixa; 2,7 a 4,4 média; 4,5 a 6,5 alta e 6,6 ou mais muito alta (Agnelli, 2015; Ministério da Saúde [MS], 2012).

Ao observar os dados do levantamento nacional Saúde Bucal Brasil 2010 (MS, 2012), o CPOD médio no Brasil foi de 16,75 na faixa etária de 35 a 44 anos e de 27,53 na faixa de 65 a 74 e destaca-se o componente perdido com 44,7\% do índice no grupo de 35 a 44 anos e o componente cariado com 8,8\%. No grupo de 65 a 74 anos o componente perdido corresponde a 91,9\% e o cariado com 1,9\%, esse contexto nacional corrobora com o estudo de Araújo et al (2009) que registrou altos índices de CPOD e altas taxas de dentes perdidos nos pacientes com câncer.

Hong et al. (2010), avaliaram a compreensão atual do impacto das terapias de câncer na cavidade oral (dentes e periodonto) encontrando um maior índice CPOD nos pacientes pós-radioterapia, e casos de infecção dentária em pacientes submetidos à quimioterapia.

No relato em questão, o paciente parcialmente dentado apresentava restos radiculares e cáries dentárias confirmando com a situação buco-dental encontrada nos estudos relatados, sendo detectada uma prevalência de dentes perdidos e cariados, refletindo uma condição de saúde bucal insatisfatória com consequente impacto negativo na qualidade de vida do paciente podendo, inclusive, comprometer o tratamento antineoplásico. Foi realizada a adequação buco-dental através de exodontias múltiplas, contribuindo para o elevado número do CPOD e para a necessidade de uso de prótese dentária. O edentulismo é um tipo de agravo de saúde bucal que repercute na saúde geral do indivíduo e representa a gravidade da situação buco-dental da população, interferindo diretamente na estética, na fonética e na função mastigatória. Além disso, sua alta prevalência em alguns grupos populacionais refletem um serviço de saúde que oferece, principalmente, um atendimento odontológico curativo, mutilador, de baixa cobertura e baixo impacto epidemiológico (Ferreira et al., 2021).

De acordo com Araújo et al. (2009), essas questões abordadas podem ser elucidadas pela dificuldade de acesso da maior parte da população brasileira ao serviço de saúde bucal, o que evidencia a falta de cuidados prévios de higiene oral e assistência odontológica preventiva, conservadora no âmbito das assistências primária e secundária; e a necessidade de uma maior integração entre os níveis de atenção para uma atenção odontológica mais integral e responsável.

É importante ressaltar que mais de $80 \%$ dos problemas buco-dentais poderiam ser solucionados na atenção primária. Porém, quando isto não acontece e o indivíduo necessita de atendimento de alta complexidade o profissional se depara com os 
agravos da doença como a cárie dentária e a doença periodontal, favorecendo infecções e óbitos (Ministério da saúde [MS], 2009).

Diante disso, como descrito no caso clínico o paciente foi encaminhado ao serviço de odontologia - CEON/HUOC/UPE anteriormente ao início da terapia antineoplásica para adequação do meio bucal através da eliminação dos focos de infecção, pois de acordo com o Protocolo Operacional Padrão de Cuidados Orais (POP-Oral) (Vidal, 2009) é necessário a realização do exame clínico minucioso previamente ao tratamento do câncer, avaliação das condições dentárias e periodontais, pois os processos infecciosos e inflamatórios devem ser eliminados. É importante evidenciar que o paciente deve adquirir hábitos de higiene bucal adequado para que possa conter a placa bacteriana durante e após o tratamento antineoplásico (Macedo et al., 2019).

No relato de caso retratado o paciente foi submetido a um primeiro protocolo de cuidados orais assim que iniciou a quimioterapia para evitar e/ou minimizar o surgimento e intensidade dos efeitos colaterais na cavidade oral. Apesar da adequação buco-dental e instituição do protocolo de cuidados orais (POP-Oral), o paciente apresentou quadro de xerostomia, disgeusia, mucosite oral grau II (Escala OMS/WHO), dor oral e exposição óssea em rebordo alveolar superior esquerdo; exemplificando a necessidade de cuidado buco-dental especializado e a possibilidade de controle dos efeitos adversos orais, decorrentes da ação dos quimioterápicos e da radioterapia em região de cabeça e pescoço realizados para o tratamento do câncer.

No estudo realizado por Neves (2018), as complicações que mais acometeram os pacientes foram a mucosite grau I, principalmente pacientes masculinos de 31 a 60 anos. A xerostomia, foi a segunda complicação mais prevalente, não apresentando diferença entre os sexos. A candidíase, disfagia e trismo também estiveram presentes. Com isso, foi possível verificar que os pacientes submetidos à quimioterapia ficaram mais susceptíveis as complicações orais agudas (Neves, 2018).

A mucosite oral apresenta-se em graus variados de eritema podendo evoluir para ulcerações (Borges et al., 2018). A mucosite oral grave pode acarretar a necessidade de nutrição enteral ou parenteral e analgésicos favorecendo infecções sistêmicas pelo fato da barreira da mucosa oral está rompida (Hong et al., 2019). A laserterapia está preconizada como principal ferramenta no manejo da mucosite, cuja aplicação poderá ser diária, propiciando conforto e qualidade de vida até que haja a remissão dos sintomas (Vidal, 2015). O uso do laser de baixa potência é utilizado para prevenir o surgimento de mucosite oral, pois apresenta capacidade de bioestimular e aumentar o metabolismo celular evitando o aparecimento das lesões na cavidade oral, promove a cicatrização e contribui também para a analgesia. Além do laser, outras substâncias para bochechos e medicamentos de ação tópica são utilizadas para controle de dor e inflamação (Borges et al., 2018; Macedo et al., 2019).

A disgeusia acontece pela atrofia das papilas gustativas ocasionadas pela radiação, a xerostomia ocorre pela disfunção das glândulas salivares e a osteorradionecrose se caracteriza pela exposição de osso desvitalizado através de uma abertura na pele ou mucosa, sendo o tratamento: prescrição de bochechos de solução aquosa de gluconato de clorexidina a $0,12 \%$ associado à higiene oral, remoção de sequestros ósseos e possíveis irritantes locais (Borges et al., 2018).

Os protocolos de prevenção são iguais aos utilizados no tratamento das complicações, porém em distintos protocolos de aplicação (Macedo et al., 2019). A partir disso, o paciente foi submetido a um ajuste de protocolo, sendo instituído o Protocolo Operacional Padrão de Cuidados Orais (POP-Oral) para o tratamento de necrose óssea, posteriormente o paciente recuperou a mucosa oral e seguiu em acompanhamento para a reabilitação oral protética.

A fim de oferecer uma melhor qualidade de vida ao paciente oncológico é preciso um tratamento multidisciplinar, pois é essencial o acompanhamento desses pacientes por médicos, cirurgiões-dentistas, fonoaudiólogos, nutricionistas e psicólogos (Arantes et al., 2020). O cirurgião-dentista deve fazer o acompanhamento desses pacientes antes, durante e após o tratamento 
do câncer, para que providências sejam tomadas para controlar, minimizar, evitar e prevenir complicações bucais que possam acometer esses pacientes (Macedo et al., 2019).

\section{Considerações Finais}

O caso clínico relatado apresenta sucesso em seu plano de tratamento, no qual tem como objetivo a prevenção e o controle das complicações orais em paciente oncológico. Destacando a importância do acompanhamento especializado e a integração do cirurgião-dentista na equipe oncológica multiprofissional antes, durante e após o tratamento antineoplásico, possibilitando ao paciente um atendimento integral e humanizado. Registra-se a necessidade imperiosa de melhor assistência e resolutividade odontológica nos níveis primário e secundário de atenção à saúde, a fim de minimizarmos o edentulismo, o qual persiste como um grave problema de saúde pública.

\section{Referências}

Agnelli, P. (2015). Variação do índice CPOD do Brasil no período de 1980 a 2010. Revista Brasileira de Odontologia, 72(1/2), 10-15.

Albrecht, J., Meves, A. \& Bigby, M. (2005). Case reports and case series from Lancet had significant impact on medical literature. J Clin Epidemiol, 58, 12271232 .

Arantes, B., Loiola, T., Garcia, N., \& Favretto, C. (2020). Manifestações bucais em paciente submetido à quimioterapia e radioterapia na região de cabeça e pescoço-relato de caso clínico. Revista saúde multidisciplinar, 8(2).

Araújo, S. S., Padilha, D. M., \& Baldisserotto, J. (2009). Avaliação da condição de saúde bucal e da qualidade de vida de pacientes com câncer de cabeça e pescoço atendidos em um hospital público de Porto Alegre. Revista Brasileira de Cancerologia, 55(2), 129-138.

Bezerra, P.A., Oliveira, M. E., Gomes, T., Barbosa, J., \& Medeiros, E. (2015). Principais Patologias Orofaciais Recorrentes em Serviços de Urgências Odontológicas: Uma Revisão da Literatura. Revista de Psicologia, 9(27), 167-177.

Borges, B., Vale, D., Aoki, R., Trivino, T., \& Fernandes, K. (2018). Atendimento odontológico de paciente submetido à radioterapia em região de cabeça e pescoço: relato de caso clínico. Revista de Odontologia da Universidade Cidade de São Paulo, 30(3), 332-340.

Calmon, M., Musso, M. A., Dell'antonio, L., Zandonade, E., Amorim, M. H., \& Miotto, M. H. (2019). Impact of oral health problems on the quality of life of women with breast cancer. Revista Gaúcha de Odontologia, 67.

Cândido, E., Ribeiro, T. R., Brito, M. A., Rodrigues, S., Feitosa, M. D., Feitosa, F., \& Freitas, J. (2021). Ethical aspects and procedures for the publication of case reports in Brazil. Research, Society and Development, 10(2).

Domingos, P., Passalacqua, M. L., \& Oliveira, A. L. (2014) Câncer bucal: um problema de saúde pública. Revista de Odontologia da Universidade Cidade de São Paulo, 26(1), 46-52.

Duarte Filho, E. S., Silva, P. F., Donato, L.F., Frigo, L., Youssef, M. N., \& Ferreira, S. J. (2019). Cárie de radiação: efeitos da radioterapia na estrutura dentária. Revista Cubana de Estomatologia, 56(1), 86-92.

Estrela, C. (2018). Metodologia Científica: Ciência, Ensino, Pesquisa (3a ed.). Editora Artes Médicas.

Ferreira Filho, M. J., Alves, D., Câmara, I., Souza, Q. H., Brasil, S., Aguiar, J., Milério, L. R., \& Mousinho, L. (2021). Reabilitação oral com prótese parcial removível dupla: revisão de literatura. Brazilian Journal Of Development, 7(2), 16934-16947.

Figueiredo, P., \& Nogueira, A. J. (2013). Prevalência de Neoplasias, Cárie e Gengivite em Pacientes Oncológicos Pediátricos no Município de Belém, Pará. Pesquisa Brasileira em Odontopediatria e Clínica Integrada, 13(2), 141-146.

Floriano, D., Ribeiro, P. F., Maragno, A. C., Rossi, K., \& Simões, P. W. (2017). Complicações orais em pacientes tratados com radioterapia ou quimioterapia em um hospital de Santa Catarina. Revista de Odontologia da Universidade Cidade de São Paulo, 29(3), $230-236$.

Ghelardi, I. R., Soares Junior, L. A., Santos, P. S., Teixeira, S., Matsushita, C., Carrillo, C., Antequera, R., \& Peres, M. P. (2008). A necessidade da avaliação e tratamento odontológico pré-radioterapia. Prática Hospitalar, 58, 149-151.

Hespanhol, F. L., Tinoco, E., Teixeira, H. G., Flabella, M. E., \& Assis, N. M. (2010). Manifestações bucais em pacientes submetidos à quimioterapia. Ciência \& Saúde Coletiva, 15, 1085-1094.

Hong, C. H., Napeñas, J. J., Hodgson, B. D., Stokman, M. A., Mathers-Stauffer, V., Elting, L. S., Spijkervet, F. K., Brennan, M. T., \& Dental Disease Section, Oral Care Study Group, Multi-national Association of Supportive Care in Cancer (MASCC)/International Society of Oral Oncology (ISOO) (2010). A systematic review of dental disease in patients undergoing cancer therapy. Supportive care in cancer: official journal of the Multinational Association of Supportive Care in Cancer, 18(8), 1007-1021.

Hong, C., Gueiros, L. A., Fulton, J. S., Cheng, K., Kandwal, A., Galiti, D., Fall-Dickson, J. M., Johansen, J., Ameringer, S., Kataoka, T., Weikel, D., Eilers, J., Ranna, V., Vaddi, A., Lalla, R. V., Bossi, P., Elad, S., \& Mucositis Study Group of the Multinational Association of Supportive Care in Cancer/International 
Research, Society and Development, v. 10, n. 15, e57101522530, 2021

Society for Oral Oncology (MASCC/ISOO) (2019). Systematic review of basic oral care for the management of oral mucositis in cancer patients and clinical practice guidelines. Supportive care in cancer: official journal of the Multinational Association of Supportive Care in Cancer, $27(10), 3949-3967$.

Instituto Nacional de Câncer José Alencar Gomes da Silva. (2019). Estimativa 2020: incidência de câncer no Brasil.

Lakatos, E. M. \& Marconi, M. A. (2003). Fundamentos de metodologia científica (5a ed.). Atlas.

Macedo, T., Melo, M.C., \& Vidal, A.K. (2019). Assistência odontológica hospitalar e oncológica: uma série de casos. Revista Gaúcha de Odontologia., 67.

Ministério da Saúde. (2009). Serviço de Odontologia em Oncologia (2 a ed.). Secretaria de Atenção à Saúde, Instituto Nacional do Câncer, Rotinas Internas do INCA.

Ministério da Saúde. (2012). Saúde Bucal Brasil 2010 Pesquisa Nacional de Saúde Bucal: resultados principais. Secretaria de Atenção à Saúde, Secretaria de Vigilância em Saúde.

Munerato, M. C., Flaminchi, D. L., \& Pethy, P. C. (2005). Urgências em Odontologia: Um Estudo Retrospectivo. Revista da Faculdade de Odontologia, 46(1), 90- 95

Neves, L. E. (2018). Prevalência Das Complicações Orais Agudas Em Pacientes Internados Em Tratamento Antineoplásico. [Trabalho de conclusão de curso, Universidade Estadual da Paraíba].

Osterne, R., Brito, R., Nogueira, R. L., Soares, E., Alves, A. P., Moura, J. F., Holanda, R., \& Sousa, F. (2008). Saúde Bucal em pacientes portadores de neoplasias malignas: Estudo clínico - Epidemiológico e Análise de Necessidades Odontológicas de 421 pacientes. Revista Brasileira de Cancerologia, 54(3), 221-226.

Paiva, M. D., Biase, R. C., Moraes, J. J., Ângelo, A., \& Honorato, M. C. (2010). Complicações Orais Decorrentes da Terapia Antineoplásica. Arquivos em Odontologia, 46(1), 48-55.

Pereira, A. S., Shitsuka, D. M., Pereira, F. J. \& Shitsuka, R. (2018). Metodologia da pesquisa científica. UFSM.

Queiroz, M., Verli, F., Marinho, S. A., Paiva, P. C., Santos, S. M., \& Soares, J. (2019). Dor, ansiedade e qualidade de vida relacionada à saúde bucal de pacientes atendidos no serviço de urgência odontológica. Ciência \& Saúde Coletiva, 24(4), 1277-1286.

Tosin, D. C. (2017). Tratamento odontológico realizado em pacientes com diagnóstico de câncer atendidos no Serviço de Odontologia Oncológica da UNACON do Hospital Geral de Palmas/Tocantins, no período de abril de 2011 a dezembro de 2016. [Dissertação de Mestrado, Faculdade de Odontologia da Universidade de São Paulo].

Vidal, A. K. (2009). Protocolo Operacional Padrão de Cuidados Buco-Dentais (POP- Oral) para indivíduos sob terapia antineoplásica. Adaptado das Rotinas Internas do INCA (serviço de Odontologia).

Vidal, A. K. (2015). Importância da Odontologia para o paciente oncológico. In C. Marques, C. Barreto, V. L. Morais, \& N. Júnior (Org.), Oncologia: Uma abordagem Multidisciplinar (pp. 775-788). Carpe Diem.

Vieira, D., Leite, A., Melo, N., \& Figueiredo, P. T. (2012). Tratamento odontológico em pacientes oncológicos. Oral Sciences, 4(2), 37-42. 